\title{
Regularity for solutions of the total variation denoising problem
}

\section{Vicent Caselles, Antonin Chambolle and Matteo Novaga}

\begin{abstract}
The main purpose of this paper is to prove a local Hölder regularity result for the solutions of the total variation based denoising problem assuming that the datum is locally Hölder continuous. We also prove a global estimate on the modulus of continuity of the solution in convex domains of $\mathbb{R}^{N}$ and some extensions of this result for the total variation minimization flow.
\end{abstract}

\section{Introduction}

We study the local regularity properties of a local minimizer of the functional

$$
\int_{\Omega}|D u|+\frac{\lambda}{2} \int_{\Omega}|u(x)-f(x)|^{2} d x
$$

where $\Omega$ is an open set in $\mathbb{R}^{N}, \lambda>0$, and $f: \Omega \rightarrow \mathbb{R}$ is locally Hölder continuous. Our main purpose is to prove that $u$ is also locally Hölder continuous (with the same exponent).

The previous functional was introduced as a model for image denoising by Rudin, Osher, and Fatemi in [22]. In that context, $\Omega$ is a bounded domain and $f$ represents the observed image which we assume to be related to the undistorted image by

$$
f=u+n,
$$

where $n$ represents a Gaussian white noise of zero mean and standard deviation $\sigma$. The parameter $\lambda>0$ may be interpreted as a regularization parameter or as a Lagrange multiplier in order to adjust the constraint $\int_{\Omega}(u-f)^{2} d x \leq|\Omega| \sigma^{2}$ determined by (1.2). 
One of the main features of total variation denoising (1.1), confirmed by numerical experiments, is its ability to restore the discontinuities of the image $[22,13,14]$. The underlying a priori assumption is that functions of bounded variation (the $B V$ model [3]) are a reasonable functional model for many problems in image processing, in particular, for denoising and restoration problems. Typically, functions of bounded variation admit a set of discontinuities which is countably rectifiable [3], being continuous in some sense (in the measure theoretic sense) away from discontinuities. The discontinuities could be identified with edges. The ability of total variation regularization to recover edges is one of the main features which advocates for the use of this model which had a strong influence in image processing (its ability to describe textures is less clear, even if some textures can be recovered, up to a certain scale of oscillation).

Motivated by the experimental evidence in image processing, we initiated the study of the local regularity properties of (1.1) in [12] where we proved that for any $f \in B V(\Omega) \cap L^{\infty}(\Omega)$ the set of jumps of $u$ (in the BV sense) is contained in the set of jumps of $f$. In other words, model (1.1) does not create any new discontinuity besides the existing ones. This has to be combined with results describing which discontinuities are preserved. No general statement in this sense exists but many examples are described in the papers $[8,9,2]$ and the book [5]. The preservation of a jump discontinuity depends on the curvature of the level line at the given point, the size of the jump and the regularization parameter $\lambda$. The examples support the idea that total variation may be a reasonable model in order to restore discontinuities.

In the present work we continue our analysis of the local regularity properties of (1.1) by proving that if the datum $f$ is locally Hölder continuous with exponent $\beta \in(0,1]$ in some region $\Omega^{\prime} \subset \Omega$, then its local minimizer $u$ is also locally Hölder continuous in $\Omega^{\prime}$ with the same exponent.

Recall that a function $u \in B V(\Omega)$ is a local minimizer of (1.1) if for any $v \in B V(\Omega)$ such that $u-v$ has support in a compact subset $K \subset \Omega$, we have

$$
\int_{K}|D u|+\frac{\lambda}{2} \int_{K}|u(x)-f(x)|^{2} d x \leq \int_{K}|D v|+\frac{\lambda}{2} \int_{K}|v(x)-f(x)|^{2} d x .
$$

It follows [11] that $u$ satisfies the equation

$$
-\frac{1}{\lambda} \operatorname{div} z+u=f
$$

with $z \in L^{\infty}\left(\Omega, \mathbb{R}^{N}\right)$ with $\|z\|_{\infty} \leq 1$, and $z \cdot D u=|D u|$ (see Section 2).

As in [12], our analysis of the regularity of the local minimizers of $u$ will be based on the following observation: for any $t \in \mathbb{R}$, the level sets $\{u>t\}$ 
(resp., $\{u \geq t\}$ ) are the minimal (resp., the maximal) solutions to the prescribed curvature problem

$$
\min _{E \subseteq \Omega} \operatorname{Per}(E, \Omega)+\lambda \int_{E}(t-f(x)) d x
$$

(whose solution is defined in the class of finite-perimeter sets and hence up to a Lebesgue-negligible set). The local regularity of $u$ can be described in terms of the distance of any two of its level sets. This is the main idea in [12] which is further refined here. We proved in [12] that, outside the jump discontinuities of $f$ (modulo an $\mathcal{H}^{N-1}$-null set), any two level sets at different heights cannot touch and hence the function $u$ is continuous there. To be able to assert a Hölder type regularity property for $u$ we need to prove a local estimate of the distance of the boundaries of two level sets. This will be done here under the assumption of local Hölder regularity for $f$.

Let us describe the plan of the paper. In Section 2 we recall some basic facts about functions of bounded variation. In Section 3 we collect some basic regularity results when $f \in L^{N}(\Omega)$. In Section 4 we prove the main result of the paper, namely the local Hölder regularity of the local minimizers of (1.1) in any subdomain $\Omega^{\prime}$ of $\Omega$ when the datum $f$ is locally Hölder continuous in $\Omega^{\prime}$. We also consider in Section 5 the case of global regularity of solutions of (1.4) with Neumann boundary conditions in convex domains of $\mathbb{R}^{N}$ and we then extend this result to the case of the total variation flow.

\section{Notation and preliminaries on $B V$ functions}

Let $\Omega$ be an open subset of $\mathbb{R}^{N}$. A function $u \in L^{1}(\Omega)$ whose gradient $D u$ in the sense of distributions is a (vector-valued) Radon measure with finite total variation in $\Omega$ is called a function of bounded variation. The class of such functions will be denoted by $B V(\Omega)$. The total variation of $D u$ on $\Omega$ turns out to be

$$
\sup \left\{\int_{\Omega} u \operatorname{div} z d x: z \in C_{0}^{\infty}\left(\Omega ; \mathbb{R}^{N}\right),|z(x)| \leq 1 \forall x \in \Omega\right\},
$$

where for a vector $v=\left(v_{1}, \ldots, v_{N}\right) \in \mathbb{R}^{N}$ we set $|v|^{2}:=\sum_{i=1}^{N} v_{i}^{2}$, and will be denoted by $|D u|(\Omega)$ or by $\int_{\Omega}|D u|$. The map $u \rightarrow|D u|(\Omega)$ is $L_{l o c}^{1}(\Omega)$-lower semicontinuous. $B V(\Omega)$ is a Banach space when endowed with the norm $\|u\|:=\int_{\Omega}|u| d x+|D u|(\Omega)$.

A measurable set $E \subseteq \Omega$ is said to be of finite perimeter in $\Omega$ if (2.1) is finite when $u$ is substituted with the characteristic function $\chi_{E}$ of $E$. The perimeter of $E$ in $\Omega$ is defined as $\operatorname{Per}(E, \Omega):=\left|D \chi_{E}\right|(\Omega)$. We denote by $\mathcal{L}^{N}$ and $\mathcal{H}^{N-1}$, respectively, the $N$-dimensional Lebesgue measure and the $(N-1)$-dimensional Hausdorff measure in $\mathbb{R}^{N}$. 
In case $E$ is a set with finite perimeter, one can define its "reduced boundary" $\partial^{*} E$ as the set of points where there exists the limit

$$
\lim _{\rho \rightarrow 0} \frac{D \chi_{E}\left(B_{\rho}(x)\right)}{\left|D \chi_{E}\left(B_{\rho}(x)\right)\right|}=\nu_{E}(x) \in \mathbb{S}^{N-1}
$$

and it has norm one. Then, one has the representation

$$
D \chi_{E}=\nu_{E}(x)\left|D \chi_{E}\right| \text { and }\left|D \chi_{E}\right|=\mathcal{H}^{N-1}\left\llcorner\partial^{*} E .\right.
$$

The first equality just follows from Besicovitch's derivation theorem for Radon measures, while the second from a careful study of the reduced boundary, see [16] and Section 3 in [3]. In particular, it follows that for any open set $A \subset \Omega$,

$$
\operatorname{Per}(E, A)=\mathcal{H}^{N-1}\left(\partial^{*} E \cap A\right) .
$$

If $E \subseteq \mathbb{R}^{N}$ is a measurable set and $x \in \mathbb{R}^{N}$, we define the (Lebesgue) upper density of $E$ at $x$ by

$$
\bar{D}(E, x):=\limsup _{\rho \rightarrow 0} \frac{\left|E \cap B_{\rho}(x)\right|}{\left|B_{\rho}(x)\right|}
$$

while the density, when it exists, is simply the $\operatorname{limit}_{\lim _{\rho \rightarrow 0}} E \cap B_{\rho}(x)|/| B_{\rho}(x) \mid$. Then it can also be shown that up to $\mathcal{H}^{N-1}$-negligible sets, the reduced boundary $\partial^{*} E$ of a set with finite perimeter coincides with the set of points where $E$ has density exactly $1 / 2$, and with the set of points where $E$ has density neither 0 nor 1 .

Given $u \in B V(\Omega)$, we define

$$
u^{+}(x):=\inf \{t: \bar{D}(\{u>t\}, x)=0\} \text { and } u^{-}(x):=\sup \{t: \bar{D}(\{u<t\}, x)=0\} .
$$

Then, we say that $u$ is approximately continuous at $x \in \Omega$ if and only if $u^{+}(x)=u^{-}(x)$. The set of points where $u$ is not approximately continuous is called the singular set of $u$ and denoted by $S_{u}$. If $u$ is the characteristic $\chi_{E}$ of a set with finite perimeter, then $\partial^{*} E \subset J_{\chi_{E}}$ and $\mathcal{H}^{N-1}\left(J_{\chi_{E}} \backslash \partial^{*} E\right)=0$. On the other hand, almost any level $\{u>t\}$ of a $B V$ function has finite perimeter, and there holds the co-area formula

$$
|D u|(\Omega)=\int_{-\infty}^{+\infty} \operatorname{Per}(\{u>t\}, \Omega) d t .
$$

The property, mentioned in the introduction of this note, that the level sets $\{u>t\}$ of a minimizer of (1.1) are themselves minimizers of (1.5) is a relatively easy consequence of formula (2.2), and can be easily adapted to the local setting (to show that local minimizers which satisfy (1.3) satisfy a local version of (1.5), with perturbations with compact support).

For a comprehensive treatment of functions of bounded variation, we refer the reader to [3]. 
If $z \in L_{l o c}^{\infty}\left(\Omega, \mathbb{R}^{N}\right)$ with $\operatorname{div} z \in L_{l o c}^{p}(\Omega)$, and $w \in B V_{l o c}(\Omega) \cap L_{l o c}^{q}(\Omega)$ (with $\frac{1}{p}+\frac{1}{q}=1$ ), we define the functional $z \cdot D w: C_{0}^{\infty}(\Omega) \rightarrow \mathbb{R}$ by the formula

$$
\langle z \cdot D w, \varphi\rangle:=-\int_{\Omega} w \varphi \operatorname{div} z d x-\int_{\Omega} w z \cdot \nabla \varphi d x \quad \forall \varphi \in C_{0}^{\infty}(\Omega) .
$$

It is a Radon measure in $\Omega$, which is of course finite if $z \in L^{\infty}\left(\Omega, \mathbb{R}^{N}\right)$, $\operatorname{div} z \in L^{p}(\Omega)$ and $w \in B V(\Omega) \cap L^{q}(\Omega)$. Moreover, we have $z \cdot D w=z \cdot \nabla w d x$ for all $w \in W^{1,1}(\Omega) \cap L^{\infty}(\Omega)$

Let $\Omega \subset \mathbb{R}^{N}$ be a bounded open set with Lipschitz boundary. We denote by $\nu_{\Omega}(x)$ the outer unit normal to a point $x \in \partial \Omega$. The following integration by parts formula can be found in [6]. Let $z \in L^{\infty}\left(\Omega, \mathbb{R}^{N}\right)$ with $\operatorname{div} z \in L^{p}(\Omega)$. Then there exists a function $\left[z \cdot \nu_{\Omega}\right] \in L^{\infty}(\partial \Omega)$ satisfying $\left\|\left[z \cdot \nu_{\Omega}\right]\right\|_{L^{\infty}(\partial \Omega)} \leq$ $\|z\|_{L^{\infty}\left(\Omega ; \mathbb{R}^{N}\right)}$, and such that for any $u \in B V(\Omega) \cap L^{q}(\Omega)$ we have

$$
\int_{\Omega} u \operatorname{div} z d x+\int_{\Omega} z \cdot D u=\int_{\partial \Omega}\left[z \cdot \nu_{\Omega}\right] u d \mathcal{H}^{N-1} .
$$

\section{Basic regularity}

We recall the following density estimate, which is classical for prescribed curvature problems such as (1.5) (see for instance [1]). To simplify, in the remaining of the paper, we assume that $\lambda=1$, as this parameter plays no role in the general regularity analysis.

Proposition 3.1. Assume $f \in L^{N}(\Omega)$. Then there exists $\delta>0$ and $\rho_{0}>0$ such that if $\rho<\rho_{0}$ and $B_{\rho}(x) \subset \Omega$, then, for almost any $t \in \mathbb{R}$,

$$
\left|\{u>t\} \cap B_{s}(x)\right|>0 \forall s>0 \Rightarrow\left|\{u>t\} \cap B_{\rho}(x)\right| \geq \delta \rho^{N} .
$$

The same holds for the sets $\{u<t\},\{u \geq t\}$, and $\{u \leq t\}$.

Remark 3.2. The assumption that $f \in L^{N}(\Omega)$ is essential, as otherwise we cannot expect any regularity of the boundary of a prescribed curvature set which minimizes (1.5). In fact, as long as $f \in L^{N}(\Omega)$, one sees that if $x$ is on the essential boundary of a set $E$ which minimizes $\operatorname{Per}(E, \Omega)+$ $\int_{E} f d x$, then as $\rho \rightarrow 0, \int_{B_{\rho}(x)} f d x<<\operatorname{Per}\left(E, B_{\rho}(x)\right)$ (thanks to Hölder's inequality and the relative isoperimetric inequality) so that the perimeter term dominates and tends to provide some regularity of the minimizing set, see [21]. On the other hand, if $f \notin L^{N}(\Omega)$, one cannot hope to obtain any regularity of $\partial E$ near the points where $\lim _{\rho \rightarrow 0}\left(1 / \rho^{N}\right) \int_{B_{\rho}(x)}|f|^{N} d x \rightarrow \infty$, that is, where the field term is much larger than the perimeter, as shows the following example. Let $\left(x_{n}\right)_{n \geq 1}$ be the sequence of all rational points 
in $\Omega=(0,1)^{2}$ and $f_{\varepsilon}(x)=1-\varepsilon\left(\sum_{n} 2^{-n}\left|x-x_{n}\right|^{-(1+\delta)}\right) \notin L^{2}(\Omega)$ (while $f \in$ $L^{p}(\Omega)$ for any $\left.p<2 /(1+\delta)\right)$, where $\varepsilon>0$ and $\delta>0$ are small parameters. As $f_{\varepsilon} \rightarrow 1$ when $\varepsilon \rightarrow 0$, the minimizers $E_{\varepsilon}$ of $\operatorname{Per}(E, \Omega)+\int_{E} f_{\varepsilon} d x$ go to the emptyset $\left(\left|E_{\varepsilon}\right| \rightarrow 0\right)$ as $\varepsilon \rightarrow 0$. On the other hand, the sets $E_{\varepsilon}$ must remain dense. Indeed, since $\int_{B_{\rho}\left(x_{n}\right)} f_{\varepsilon} d x \sim-C_{n, \varepsilon} \rho^{1-\delta}$ as $\rho \rightarrow 0$ (for some constant $C_{n, \varepsilon}>0$ depending on $x_{n}$ and $\varepsilon$ ), no point $x_{n}$ can be at a positive distance from $E_{\varepsilon}$, otherwise the set $E_{\varepsilon} \cup B_{\rho}\left(x_{n}\right)$ for $\rho$ small enough would have a lower energy and we would reach a contradiction. It is clear that these sets $E_{\varepsilon}$, dense and with low volume, can have no kind of regularity. We refer to [7] for other examples of singular minimizers with $f \notin L^{N}(\Omega)$.

Proof. The idea of the proof is as follows: comparing $\{u>t\}$ with $\{u>t\} \backslash$ $B_{s}(x)$, by minimality we have (for a.e. $s>0$ small)

$$
\begin{aligned}
\mathcal{H}^{N-1}\left(B_{s}(x) \cap \partial^{*}\{u>t\}\right) & \leq \\
\leq \mathcal{H}^{N-1}\left(\partial B_{s}(x)\right. & \cap\{u>t\})+\int_{B_{s}(x) \cap\{u>t\}} f(x)-t d x .
\end{aligned}
$$

Let $h(s)=\left|B_{s}(x) \cap\{u>t\}\right|=\int_{0}^{s} \mathcal{H}^{N-1}\left(\partial B_{\tau}(x) \cap\{u>t\}\right) d \tau$ : then $h \in W^{1,1}(0, \rho)$ for some $\rho>0$, with $h^{\prime}(s)=\mathcal{H}^{N-1}\left(\partial B_{s}(x) \cap\{u>t\}\right)$ for a.e. $s$.

Now, adding $\mathcal{H}^{N-1}\left(\partial B_{s}(x) \cap\{u>t\}\right)$ to both sides of (3.2), we find for a.e. $s>0$ small:

$$
\begin{aligned}
\operatorname{Per}\left(B_{s}(x) \cap\{u>t\}\right)= & \mathcal{H}^{N-1}\left(B_{s}(x) \cap \partial^{*}\{u>t\}\right) \\
& +\mathcal{H}^{N-1}\left(\partial B_{s}(x) \cap\{u>t\}\right) \\
\leq & 2 h^{\prime}(s)+\|f-t\|_{L^{N}\left(B_{s}(x)\right)} h(s)^{1-1 / N} .
\end{aligned}
$$

By equiintegrability there exists $\rho_{0}>0$ such that if $s<\rho_{0},\|f-t\|_{L^{N}\left(B_{s}(x)\right)}$ is less than half the isoperimetric constant in $\mathbb{R}^{N}$ so that

$$
\operatorname{ch}(s)^{1-1 / N} \leq h^{\prime}(s) \text {. }
$$

We get the thesis by integrating from 0 to $\rho$.

As a consequence of Proposition 3.1, if we identify the set $E_{t}=\{u>t\}$ with the set of points where it has density one, then $E_{t}$ is an open set and

$$
\mathcal{H}^{N-1}\left(\partial E_{t} \backslash \partial^{*} E_{t}\right)=0 .
$$

Indeed, if $x \in \partial E_{t}$, then it may be approximated by points in $E_{t}$ and points in $\mathbb{R}^{N} \backslash E_{t}$. Then, by Proposition 3.1, both the upper density of $x$ in $E_{t}$ and in $\mathbb{R}^{N} \backslash E_{t}$ is positive. We deduce that $x$ belongs to the measure theoretic boundary of $E_{t}$, which is the set where $E_{t}$ has Lebesgue density neither 0 nor 1 . As this set is $\mathcal{H}^{N-1}$-equivalent to $\partial^{*} E_{t}$, (3.3) follows. 
Corollary 3.3. $u^{-}$is l.s.c. and $u^{+}$is u.s.c.

Proof. If $x \in\left\{u^{-}>t\right\}$ for some $t \in \mathbb{R}$, then there exists $t^{\prime}>t$ such that $u^{-}(x) \geq t^{\prime}$. Hence,

$$
\lim _{\rho \rightarrow 0} \frac{\left|\left\{u<t^{\prime}\right\} \cap B_{\rho}(x)\right|}{\rho^{N}}=0,
$$

which implies by the previous result that there exists $\rho>0$ small such that $B_{\rho}(x) \subset\left\{u \geq t^{\prime}\right\}$, up to a negligible set. Hence, $u^{-} \geq t^{\prime}>t$ on $B_{\rho}(x)$, so that $\left\{u^{-}>t\right\}$ is open, and $u^{-}$is l.s.c.. The statement for $u^{+}$follows at once, since $u^{+}=-(-u)^{-}$.

In particular, it follows that $u$ is continuous out of $S_{u}=\{x \in \Omega$ : $\left.u^{-}(x)<u^{+}(x)\right\}$. We can give a more precise statement:

Proposition 3.4. For any $\beta \in[0,1)$, let $A_{\beta}$ be the set of points where $u$ is not $\beta$-Hölder continuous at $x$. Then, it holds

$$
\operatorname{dim}_{\mathcal{H}}\left(A_{\beta}\right) \leq N-1+\beta
$$

Proof. Let $B_{2 \rho}(x) \subset \Omega$, with $\rho<\rho_{0}$. For almost any $s \in\left(\inf _{B_{\rho}(x)} u, \sup _{B_{\rho}(x)} u\right)$, there is some $y \in B_{\rho}(x)$ such that $\left|\{u>s\} \cap B_{r}(y)\right|>0$ for all $r>0$. Then, by Proposition 3.1, we have that $\left|\{u>s\} \cap B_{r}(y)\right| \geq \delta r^{N}$ for all $r>0$. Hence $\left|\{u>s\} \cap B_{2 \rho}(y)\right| \geq \delta \rho^{N}$ and we deduce that

$$
\begin{aligned}
\operatorname{osc}_{B_{\rho}(x)}(u) & \leq \frac{1}{\delta \rho^{N}} \int_{\inf _{B_{\rho}(x)} u}^{\sup _{B_{\rho}(x)} u} \min \left\{\left|B_{2 \rho(x)} \cap\{u>s\}\right|,\left|B_{2 \rho(x)} \cap\{u<s\}\right|\right\} d s \\
& \leq \frac{C}{\rho^{N-1}} \int_{\inf _{B_{\rho}(x)} u}^{\sup _{B_{\rho}(x)} u} \operatorname{Per}\left(B_{2 \rho(x)} \cap\{u>s\}\right) d s \leq \frac{C|D u|\left(B_{2 \rho}(x)\right)}{\rho^{N-1}} .
\end{aligned}
$$

where the relative isoperimetric inequality was used in the second inequality. The thesis follows from (3.4) and [3, Th. 2.56].

In particular, it follows again from (3.4) that $u$ is continuous at each $x$ such that

$$
\frac{|D u|\left(B_{2 \rho}(x)\right)}{\rho^{N-1}} \rightarrow 0
$$

as $\rho \rightarrow 0$.

We recall that in [12], it is proven that in dimension $N \leq 7$, if $f$ is continuous in $\Omega$, then also $u$ is. We will try now to extend this result to slightly higher regularity. It is clear, though, that the highest possible regularity is Lipschitz. This can be shown by trivial examples, for instance in $1 \mathrm{D}$ if $\Omega=[-1,1], f=\gamma|x|^{2} / 2, \gamma$ large enough, and $u$ is the global minimizer of (1.1). 


\section{Interior regularity of solutions}

We will now show the local regularity of the function $u$, at least in dimension $N \leq 7$, whenever $f$ is regular. By regular, we mean either Hölder with some exponent $\beta$, or Lipschitz-continuous $(\beta=1)$. Our proofs could be adapted to more general moduli of continuity.

The proof relies on an "exclusion" principle for the level sets of $u$, which is valid near any sufficiently regular level line. However, in order to make this argument uniform, we need quite strong results of regularity for solutions of the prescribed curvature problem (i.e., the problem which our level lines satisfy). The restriction on the dimension is due to these results and the existence of singular minimal cones in dimension 8 or more. We believe, however, that this is technical and that the regularity of $u$ should be preserved near the possible singular points of the level lines.

\subsection{Local regularity of the level sets of $u$}

Let us first quote the following theorem of I. Tamanini, which is shown in [23] and relies on the previous works of Massari [18, 19], and Massari and Pepe [20]:

Theorem 4.1. [23, Theorem 1] Let $\Omega$ be an open subset of $\mathbb{R}^{N}, N \geq 2$, and let $E$ be a set with finite perimeter satisfying for $\alpha \in(0,1)$ :

$$
\psi\left(E, B_{\rho}(x)\right):=\left|D \chi_{E}\right|\left(B_{\rho}(x)\right)-\inf _{F \triangle E \Subset B_{\rho}(x)}\left|D \chi_{F}\right|\left(B_{\rho}(x)\right) \leq c \rho^{N-1+2 \alpha}
$$

for every $x \in \Omega$ and every $\rho \in(0, R)$ with $c$ and $R$ local positive constants.

Then the reduced boundary $\partial^{*} E$ is a $C^{1, \alpha}$-hypersurface in $\Omega$, and $\mathcal{H}^{s}((\partial E \backslash$ $\left.\left.\partial^{*} E\right) \cap \Omega\right)=0$ for any $s>N-8$.

Moreover, assuming that (4.1) holds uniformly for any $E_{h}$, with $\left(E_{h}\right)_{h \geq 1}$ locally convergent in $\Omega$ to some limit set $E_{\infty}$ as $h \rightarrow \infty$, we have that if $x_{h} \in \partial E_{h}$ for every $h$, with $\left(x_{h}\right)_{h \geq 1}$ convergent to some point $x_{\infty} \in \Omega$, then $x \in \partial E_{\infty}$; while, if $x_{\infty} \in \partial^{*} E_{\infty}$, then there exists $\bar{h}$ such that $x_{h} \in \partial^{*} E_{h}$ for every $h \geq \bar{h}$, and the unit outward normal to $\partial E_{h}$ at $x_{h}$ converges to the unit outward normal to $\partial E_{\infty}$ at $x_{\infty}$.

Here, "locally convergent" means that $\chi_{E_{h}} \rightarrow \chi_{E_{\infty}}$ in $L_{l o c}^{1}(\Omega)$.

Consider now a solution $u$ of (1.4) and assume $f \in L^{p}(\Omega)$ with $p>N$. Let $t \in \mathbb{R}, E_{t}=\{u>t\}$ and let $x \in \Omega$. If $\rho>0$ and $F \triangle E_{t} \Subset B_{\rho}(x)$, then

$$
\left|D \chi_{E_{t}}\right|\left(B_{\rho}(x)\right)+\int_{E_{t}}(t-f(x)) d x \leq\left|D \chi_{F}\right|\left(B_{\rho}(x)\right)+\int_{F}(t-f(x)) d x
$$


and we deduce easily

$$
\psi\left(E_{t}, B_{\rho}(x)\right) \leq \int_{B_{\rho}(x)}|t-f(x)| d x \leq\|t-f\|_{L^{p}\left(B_{\rho}(x)\right)}\left|B_{\rho}(x)\right|^{1-1 / p} .
$$

Hence, (4.1) holds for $E_{t}$ in $B_{\rho}(x)$, for $\alpha=(1-N / p) / 2$. Moreover, this estimate is uniform in $x$. We deduce the following corollary:

Corollary 4.2. Let $f \in L^{p}(\Omega), p>N$, and let $\alpha=(1-N / p) / 2$. Let $u$ solve (1.4) and, for any $t \in \mathbb{R}$, let $E_{t}=\{u>t\}$. Let $\bar{x} \in \partial^{*} E_{t}$. Then, there exists an open neigborhood $A$ of $\bar{x}$ such that for any $s \in \mathbb{R}, \partial E_{s} \cap A$ is a $C^{1, \alpha}$ hypersurface, moreover, one may assume that $\nu_{E_{s}}(x) \cdot \nu_{E_{t}}(\bar{x}) \geq \sqrt{2} / 2$ for any $x \in \partial E_{s} \cap A$.

Proof. If the corollary were not true, there would exist a sequence $x_{h}$, $x_{h} \rightarrow \bar{x}$, such that either $x_{h} \in \partial E_{t} \backslash \partial^{*} E_{t}$ (the singular set) for all $h$ large, or such that $\nu_{E_{u\left(x_{h}\right)}}\left(x_{h}\right) \cdot \nu_{E_{t}}(\bar{x})<\sqrt{2} / 2$ for all $h$ large. This would contradict Theorem 4.1.

Corollary 4.3. Let $t \in \mathbb{R}, \bar{x} \in \partial^{*} E_{t}$ and $A$ be the set given by Corollary 4.2. Choose a system of coordinates such that the last component $x_{N}$ is along the vector $\nu_{E_{t}}(\bar{x})$. Then, for any $R$, and $x^{0} \in A$, if we let $C_{R}$ be the cylinder $C_{R}=B_{R}^{\prime} \times(-R, R)=\left\{x: \sum_{i=1}^{N-1}\left(x_{i}-x_{i}^{0}\right)^{2} \leq R^{2},-R \leq x_{N}-x_{N}^{0} \leq R\right\}$, then if $C_{R} \subset A, E_{u\left(x^{0}\right)} \cap C_{R}$ is the supergraph $\left\{x_{N}>v\left(x_{1}, \ldots, x_{N-1}\right)\right\}$ of a 1-Lipschitz (and $C^{1, \alpha}$ ) function $v: B_{R}^{\prime} \rightarrow[-R, R]$.

Proof. We just need to observe that $\nu_{E_{u\left(x^{0}\right)}}$ is given by $(\nabla v,-1) / \sqrt{1+|\nabla v|^{2}}$. Hence, the condition $\nu_{E_{u\left(x^{0}\right)}}(x) \cdot \nu_{E_{t}}(\bar{x}) \geq \sqrt{2} / 2$, which holds in $A$ thanks to Corollary 4.2, becomes $1 / \sqrt{1+|\nabla v|^{2}} \geq \sqrt{2} / 2$, that is, $|\nabla v| \leq 1$.

Corollary 4.4. Under the same assumptions, assume also that $f$ is Höldercontinuous with exponent $\beta>0$ in $\Omega$ (hence, in particular, bounded, so that the above holds with any $\alpha<1$ ). Then, in addition, we have that the function $v$ in Corollary 4.3 is $C^{2, \beta}$, with a norm which does not depend on $x^{0} \in A$.

Proof. Since the graph of $v$ satisfies a mean curvature type equation with Hölder continuous right hand side, the corollary follows as a consequence of Corollary 4.3 and standard regularity results [17].

\subsection{Local regularity of $u$}

We consider here the case $f \in C^{0, \beta}(\Omega)$, with $0 \leq \beta \leq 1$ (we include the Lipschitz case for $\beta=1$ ).

Theorem 4.5. Let $N \leq 7$ and let $u$ be a solution of (1.4). Assume $f \in C^{0, \beta}$ locally in $\Omega$, for some $\beta \in[0,1]$. Then $u$ is also $C^{0, \beta}$ locally in $\Omega$. 
Proof. The idea of the proof of Theorem 4.5 is to show that two level surfaces corresponding to different values of $u$ cannot be too close one from another, because their curvature are different. In order to do so, given a level surface we build a "calibrating" vector field $\sigma$ whose flux will estimate the energy of all neighboring surfaces (and yield a contradiction whenever they come too close to the initial surface). We divide the proof into four Steps.

Step 1. The case $\beta=0$ has been treated in [12, Theorem 1 and Remark 3.4]. We therefore consider here the case where $\beta>0$. Since $N \leq 7$, all points of any level set are regular. From Corollaries 4.3 and 4.4 we know that, uniformly in the neighborhood of any regular point $x^{0} \subset\left\{u=t_{1}\right\}$ (as long as the neighborhood is inside $\Omega$ ), and after an appropriate change of coordinates, the set $\left\{u>t_{1}\right\}$ is the supergraph $x_{N}>v_{1}\left(x^{\prime}\right)$, in a suitable cylinder

$$
C_{R}=B_{R}^{\prime} \times(-R, R)=\left\{x: \sum_{i=1}^{N-1}\left(x_{i}-x_{i}^{0}\right)^{2} \leq R^{2},-R \leq x_{N}-x_{N}^{0} \leq R\right\},
$$

where we have denoted $x^{\prime}=\left(x_{1}, \ldots, x_{N-1}\right)$. We can also assume that

$$
\left\|\nabla^{\prime} v_{1}\right\|_{\infty} \leq 1 \quad \text { in } B_{R}^{\prime}, \quad \text { and } \quad\left\|D^{\prime 2} v_{1}\right\|_{\infty} \leq \kappa<\infty .
$$

We have denoted the derivatives with respect to the first $N-1$ variables with a prime (")"). To simplify, we assume that $x^{0}=0$.

We denote, for $p \in \mathbb{R}^{N-1}$,

$$
F(p)=\sqrt{1+|p|^{2}}
$$

(and $F^{*}(q)=\sup _{p} q \cdot p-F(p)$ its Legendre-Fenchel conjugate), and consider, for $\gamma \in(0,1)$, the function

$$
w_{\gamma}=v_{1}+(\gamma / 2)\left(R^{2}-\left|x^{\prime}\right|^{2}\right) .
$$

Then

$$
-\operatorname{div}^{\prime} \nabla F\left(\nabla^{\prime} w_{\gamma}\right) \leq t_{1}-f\left(x^{\prime}, v_{1}\left(x^{\prime}\right)\right)+C \gamma \quad \text { in } B_{R}^{\prime},
$$

where $C$ is an absolute constant (it is a bound on the second and third derivatives of $\left.F(p)=\sqrt{1+|p|^{2}}\right)$. Indeed, we have

$$
\begin{aligned}
\operatorname{div}^{\prime} \nabla F\left(\nabla^{\prime} w_{\gamma}\right) & =\operatorname{div}^{\prime} \nabla F\left(\nabla^{\prime} v_{1}\right)-\gamma \operatorname{div}^{\prime} \int_{0}^{1} D^{2} F\left(\nabla^{\prime} v_{1}-\gamma s x^{\prime}\right) \cdot x^{\prime} d s \\
& =-t_{1}+f\left(x^{\prime}, v_{1}\left(x^{\prime}\right)\right)-\gamma(\mathcal{R})
\end{aligned}
$$


where the rest $(\mathcal{R})$ is given by (the sums range from 1 to $N-1$ ):

$$
\begin{aligned}
(\mathcal{R})= & \int_{0}^{1} \partial_{i}\left(\partial_{i, j}^{2} F\left(\nabla^{\prime} v_{1}-\gamma s x^{\prime}\right) x_{j} d s\right. \\
= & \int_{0}^{1} \operatorname{Tr} D^{2} F\left(\nabla^{\prime} v_{1}-\gamma s x^{\prime}\right) d s \\
& +\int_{0}^{1} \partial_{i, j, k}^{3} F\left(\nabla^{\prime} v_{1}-\gamma s x^{\prime}\right)\left(\partial_{i, k}^{2} v_{1}-\gamma s \delta_{i, k}\right) x_{j} d s \\
\leq & \int_{0}^{1} \operatorname{Tr} D^{2} F\left(\nabla^{\prime} v_{1}-\gamma s x^{\prime}\right) d s+C R\left\|D^{\prime 2} v_{1}\right\|_{L^{\infty}\left(B_{R}^{\prime}\right)} \\
& +\int_{0}^{1} s \partial_{s}\left[\operatorname{Tr} D^{2} F\left(\nabla^{\prime} v_{1}-\gamma s x^{\prime}\right)\right] d s \\
= & C R\left\|D^{\prime 2} v_{1}\right\|_{L^{\infty}\left(B_{R}^{\prime}\right)}+\operatorname{Tr} D^{2} F\left(\nabla^{\prime} v_{1}-\gamma x^{\prime}\right) \\
\leq & C\left(R\left\|D^{\prime 2} v_{1}\right\|_{L^{\infty}\left(B_{R}^{\prime}\right)}+1\right) .
\end{aligned}
$$

Hence, using $\left|D^{\prime 2} v_{1}\right| \leq \kappa$ in $B_{R}^{\prime}$, we get a uniform bound for $(\mathcal{R})$, and we obtain (4.2).

Step 2. Now we build, in $C_{R}^{+}=\left\{x \in C_{R}: x_{N}>v_{1}\left(x^{\prime}\right)\right\}$ the vector field

$$
\sigma\left(x^{\prime}, w_{\gamma}\left(x^{\prime}\right)\right)=\left(\begin{array}{c}
\nabla F\left(\nabla^{\prime} w_{\gamma}\left(x^{\prime}\right)\right) \\
F^{*}\left(\nabla F\left(\nabla^{\prime} w_{\gamma}\left(x^{\prime}\right)\right)\right)
\end{array}\right)
$$

which is Lipschitz-continuous and has everywhere norm equal to 1 . We show that, in $C_{R}^{+}$,

$$
\operatorname{div} \sigma(x) \geq-t_{1}+f\left(x^{\prime}, v_{1}\left(x^{\prime}\right)\right)-C \gamma(x)
$$

where $\gamma(x)$ is the unique value of $\gamma$ such that $w_{\gamma}\left(x^{\prime}\right)=x_{N}$, that is, $\gamma(x)=$ $2\left(x_{N}-v_{1}\left(x^{\prime}\right)\right) /\left(R^{2}-\left|x^{\prime}\right|^{2}\right)$.

Let $\sigma=\left(\sigma_{i}\right)_{i=1}^{N}$ and $\sigma^{\prime}$ denote the first $N-1$ coordinates of $\sigma$. For fixed $\gamma>0$, we have using (4.2)

$$
\operatorname{div}^{\prime}\left[\sigma^{\prime}\left(x^{\prime}, w_{\gamma}\left(x^{\prime}\right)\right)\right]=\operatorname{div}^{\prime} \nabla F\left(\nabla^{\prime} w_{\gamma}\right) \geq-t_{1}+f\left(x^{\prime}, v_{1}\left(x^{\prime}\right)\right)-C \gamma .
$$

Observe that

$$
\operatorname{div}^{\prime}\left[\sigma^{\prime}\left(x^{\prime}, w_{\gamma}\left(x^{\prime}\right)\right)\right]=\sum_{i=1}^{N-1}\left(\partial_{i} \sigma_{i}\right)\left(x^{\prime}, w_{\gamma}\left(x^{\prime}\right)\right)+\sum_{i=1}^{N-1}\left(\partial_{N} \sigma_{i}\right)\left(x^{\prime}, w_{\gamma}\left(x^{\prime}\right)\right) \partial_{i} w_{\gamma}\left(x^{\prime}\right) .
$$

Now, for fixed $x^{\prime} \in B_{R}^{\prime}$, we have for $i=1, \ldots, N$, using the chain rule:

$$
\partial_{\gamma}\left[\sigma_{i}\left(x^{\prime}, w_{\gamma}\left(x^{\prime}\right)\right)\right]=\left(\partial_{N} \sigma_{i}\right)\left(x^{\prime}, w_{\gamma}\left(x^{\prime}\right)\right) \frac{R^{2}-\left|x^{\prime}\right|^{2}}{2} .
$$


On the other hand, using $\nabla F^{*}(\nabla F(p))=p$ for any $p \in \mathbb{R}^{N-1}$, we have

$$
\begin{aligned}
\partial_{\gamma}\left[\sigma_{N}\left(x^{\prime}, w_{\gamma}\left(x^{\prime}\right)\right)\right] & =\nabla F^{*}\left(\nabla F\left(\nabla^{\prime} w_{\gamma}\left(x^{\prime}\right)\right)\right) \cdot \partial_{\gamma}\left[\nabla F\left(\nabla^{\prime} w_{\gamma}\left(x^{\prime}\right)\right)\right] \\
& =\sum_{i=1}^{N-1} \partial_{i} w_{\gamma}\left(x^{\prime}\right) \partial_{\gamma}\left[\sigma_{i}\left(x^{\prime}, w_{\gamma}\left(x^{\prime}\right)\right)\right] \\
& =\sum_{i=1}^{N-1} \partial_{i} w_{\gamma}\left(x^{\prime}\right)\left(\partial_{N} \sigma_{i}\right)\left(x^{\prime}, w_{\gamma}\left(x^{\prime}\right)\right) \frac{R^{2}-\left|x^{\prime}\right|^{2}}{2}
\end{aligned}
$$

where we have used (4.7) in the last equality. Then, dividing by $\left(R^{2}-\right.$ $\left.\left|x^{\prime}\right|^{2}\right) / 2$, we find from (4.7) (with $i=N$ ) and the last equation that

$$
\left(\partial_{N} \sigma_{N}\right)\left(x^{\prime}, w_{\gamma}\left(x^{\prime}\right)\right)=\sum_{i=1}^{N-1} \partial_{i} w_{\gamma}\left(x^{\prime}\right)\left(\partial_{N} \sigma_{i}\right)\left(x^{\prime}, w_{\gamma}\left(x^{\prime}\right)\right) .
$$

Combining this with (4.5) and (4.6), we obtain

$$
(\operatorname{div} \sigma)\left(x^{\prime}, w_{\gamma}\left(x^{\prime}\right)\right) \geq-t_{1}+f\left(x^{\prime}, v_{1}\left(x^{\prime}\right)\right)-C \gamma,
$$

which yields (4.4).

Step 3. Let $t_{2}>t_{1}$ and consider the set $E_{2}=\left\{u>t_{2}\right\}$. Let $C_{f}$ be the Hölder constant of $f, \gamma \leq 1$ and $1-\beta \geq 0$. Let

$$
W_{\gamma}=\left\{v_{1}\left(x^{\prime}\right)<x_{N}<w_{\gamma}\left(x^{\prime}\right)\right\} .
$$

Then, we claim that $E_{2} \cap W_{\gamma}=\emptyset$ as soon as $\gamma^{\beta} \leq\left(t_{2}-t_{1}\right) /\left(C+C_{f}\left(R^{2} / 2\right)^{\beta}\right)$.

Observe that $E_{2} \cap C_{R} \subset C_{R}^{+}$. Assume that $\gamma$ is such that $E_{2} \cap W_{\gamma}$ is not empty. Then, by minimality of $E_{2}$, we have

$$
\mathcal{H}^{N-1}\left(\partial E_{2} \cap W_{\gamma}\right)+\int_{E_{2} \cap W_{\gamma}}\left(t_{2}-f(x)\right) d x \leq \mathcal{H}^{N-1}\left(\partial W_{\gamma} \cap E_{2}\right) .
$$

Using the fact that, by construction, $\sigma$ is the inner normal to $W_{\gamma}$, from Gauss-Green's formula and (4.4) we deduce

$$
\begin{aligned}
\mathcal{H}^{N-1}\left(\partial W_{\gamma} \cap E_{2}\right) & -\mathcal{H}^{N-1}\left(\partial E_{2} \cap W_{\gamma}\right) \leq \\
& \leq \int_{\partial W_{\gamma} \cap E_{2}}(-\sigma \cdot \nu) d \mathcal{H}^{N-1}+\int_{W_{\gamma} \cap \partial E_{2}}(-\sigma \cdot \nu) d \mathcal{H}^{N-1} \\
& =\int_{\partial\left(W_{\gamma} \cap E_{2}\right)}(-\sigma \cdot \nu) d \mathcal{H}^{N-1} \\
& \leq \int_{W_{\gamma} \cap E_{2}}\left(t_{1}-f\left(x^{\prime}, v_{1}\left(x^{\prime}\right)\right)+C \gamma\right) d x
\end{aligned}
$$


Hence

$$
\int_{W_{\gamma} \cap E_{2}}\left(t_{2}-f(x)-t_{1}+f\left(x^{\prime}, v_{1}\left(x^{\prime}\right)\right)-C \gamma\right) d x \leq 0
$$

and, since $\left|f\left(x^{\prime}, v_{1}\left(x^{\prime}\right)\right)-f(x)\right| \leq C_{f}\left(\gamma R^{2} / 2\right)^{\beta}$, this is impossible as soon as

$$
\left(C \gamma^{1-\beta}+C_{f}\left(\frac{R^{2}}{2}\right)^{\beta}\right) \gamma^{\beta}<t_{2}-t_{1}
$$

By continuity, and using $\gamma \leq 1$ and $1-\beta \geq 0$, we deduce that $E_{2} \cap W_{\gamma}=\emptyset$ as soon as $\gamma^{\beta} \leq\left(t_{2}-t_{1}\right) /\left(C+C_{f}\left(R^{2} / 2\right)^{\beta}\right)$.

Step 4. Conclusion. For any for any $x \in C_{R / 2}, \bar{x} \in\left\{u=t_{1}\right\} \cap C_{R / 2}$, we have

$$
|u(x)-u(\bar{x})| \leq\left(\frac{8}{3 R^{2}}\right)^{\beta}\left(C+C_{f}\left(R^{2} / 2\right)^{\beta}\right)|x-\bar{x}|^{\beta} .
$$

Assume that $u(x)=t_{2}>t_{1}$ (a symmetric construction can be done below the graph of $v_{1}$ ). By Step 3 we have that $x \in\left\{u>t_{2}^{\prime}\right\} \backslash W_{\gamma}$ where $t_{2}^{\prime}=t_{2}-\epsilon$ and $\gamma^{\beta}=\left(t_{2}^{\prime}-t_{1}\right) /\left(C+C_{f}\left(R^{2} / 2\right)^{\beta}\right)$. Then

$$
|x-\bar{x}| \geq w_{\gamma}\left(x^{\prime}\right) \geq \frac{3}{8} \gamma R^{2} .
$$

Hence

$$
|x-\bar{x}|^{\beta} \geq\left(\frac{3 R^{2}}{8}\right)^{\beta} \frac{\left|t_{2}^{\prime}-t_{1}\right|}{C+C_{f}\left(R^{2} / 2\right)^{\beta}} .
$$

Letting $t_{2}^{\prime} \rightarrow t_{2}$, this shows that in $C_{R / 2}$, the distance between $\left\{u=t_{1}\right\}$ and $\left\{u=t_{2}\right\}$ is bounded from below by $(3 / 8) R^{2}\left[\left(t_{2}-t_{1}\right) /\left(C+C_{f}\left(R^{2} / 2\right)^{\beta}\right)\right]^{1 / \beta}$, i.e., (4.8) holds.

Since $R$ and $C$ can be chosen uniform in the neighborhood $A^{\prime} \Subset \Omega$ of any open set $A \Subset \Omega$, this yields that $u$ is $\beta$-Hölder (Lipschitz, when $\beta=1$ ) in $\left\{x \in A^{\prime}: \operatorname{dist}(x, \partial A)>\sqrt{2} R\right\}$, which contains $A$ if $R$ was chosen small enough.

We are able to extend the above result to the total variation flow in case that we have a uniform convergence of the implicit in time Euler scheme. This can be proved for instance for the total variation flow with Neumann boundary conditions in convex domains and this is the purpose of our next Section. We expect the local regularity result for the total variation flow to be true in general. 


\section{Global minimizers on convex domains}

In this section we assume that $\Omega \subset \mathbb{R}^{N}$ is a convex domain.

Let $f: \bar{\Omega} \rightarrow \mathbb{R}$ be a uniformly continuous function, with modulus of continuity $\omega_{f}:[0,+\infty) \rightarrow[0,+\infty)$. We consider the solution $u$ of $(1.4)$ with homogeneous Neumann boundary condition, that is, such that (1.3) for any compact set $K \subset \bar{\Omega}$ and any $v \in B V(\Omega)$ such that $v=u$ out of $K$. This solution is unique, as can be shown adapting the proof of [11, Cor. C.2.] (see also [4] for the required adaptations to deal with the boundary condition), which deals with the case $\Omega=\mathbb{R}^{N}$.

Then, the following result holds true:

Theorem 5.1. Assume $N \leq 7$. Then, the function $u$ is uniformly continuous in $\Omega$, with modulus $\omega_{u} \leq \omega_{f}$.

Again, is quite likely here that the assumption $N \leq 7$ is not necessary for this result.

Proof. We first assume that $\Omega$ is bounded, smooth and uniformly convex. Let us also assume that $f$ is smooth up to the boundary. Let $a \in \mathbb{R}$ and consider the set $E_{a}=\{u>a\}$. Then, we now have that

$$
\operatorname{Per}\left(E_{a}, \Omega\right)+\int_{E_{a}}(a-f(x)) d x \leq \operatorname{Per}(E, \Omega)+\int_{E}(a-f(x)) d x
$$

for any finite-perimeter set $E \subset \Omega$. In particular, $\partial E_{a}$ is smooth up to the boundary, and orthogonal to $\partial \Omega$ at the contact points. Then $\partial E_{a}$ satisfies the prescribed mean curvature equation

$$
\mathbf{H}_{\partial E_{a}}=f-a \quad \text { on } \partial E_{a}
$$

where $\mathbf{H}_{\partial E_{a}}$ is the mean curvature of $\partial E_{a}$ with the convention that we oriented the surface with the outer unit normal.

Choose now $t>s$ and consider the sets $E_{t} \subseteq E_{s}$. For simplicity, let us write

$$
\tilde{\partial} E_{t}=\overline{\partial E_{t} \cap \Omega}, \quad \tilde{\partial} E_{s}=\overline{\partial E_{s} \cap \Omega} .
$$

Let

$$
\delta=\operatorname{dist}\left(\tilde{\partial} E_{t}, \tilde{\partial} E_{s}\right)=\min \left\{|x-y|: x \in \tilde{\partial} E_{t}, y \in \tilde{\partial} E_{t}\right\} \geq 0,
$$

and choose $x_{t} \in \tilde{\partial} E_{t}$ and $x_{s} \in \tilde{\partial} E_{s}$ such that $\left|x_{t}-x_{s}\right|=\delta$. We let $e$ be the outer normal to $\tilde{\partial} E_{t}$, at $x_{t}$, which is also the outer normal to $\tilde{\partial} E_{s}$ at $x_{s}$ and is given by $e=\left(x_{s}-x_{t}\right) / \delta$ whenever $\delta>0$.

Since we already know that $u$ is continuous inside $\Omega$ [12], then the equality $x_{s}=x_{t}$ could only happen in $\partial \Omega$. But this cannot happen since both 
$\partial E_{s}$ and $\partial E_{t}$ satisfy the prescribed mean curvature equation (5.2) classically up to the boundary, with Neumann boundary condition, and $t>s$. Thus we have that $\delta>0$. Notice also that, since $\Omega$ is strictly convex, none of the points $x_{s}, x_{t}$ can lie on its boundary: indeed if, for instance, we had $x_{s} \in \partial \Omega$ (and $x_{t} \in \bar{\Omega}$ ), we would have $\left(x_{t}-x_{s}\right) \cdot \nu_{\Omega}\left(x_{s}\right)<0$, and since $-\nu_{\Omega}\left(x_{s}\right)$ is tangent to $\tilde{\partial} E_{s}$, pointing towards its interior, we would contradict the minimality of $\left\|x_{t}-x_{s}\right\|$ with respect to $x_{s}$.

Let $C$ be the connected component of $\left(\tilde{\partial} E_{t}+\delta e\right) \cap \tilde{\partial} E_{s}$ containing $x_{s}$. Since $\Omega$ is strictly convex, we have that $C$ is a compact subset of $\left(x_{s}+e^{\perp}\right) \cap \Omega$. In particular, if $\varepsilon>0$ is small enough, then the open set $\left(E_{t}+(\delta+\varepsilon) e\right) \backslash \bar{E}_{s}$ has a connected component $W_{\varepsilon}$ with $C \subset \partial W_{\varepsilon}$ and which is strictly contained in $\Omega$.

We use (5.1), comparing $E_{t}$ with $E_{t} \backslash\left(W_{\varepsilon}-(\delta+\varepsilon) e\right)$ and $E_{s}$ with $E_{s} \cup W_{\varepsilon}$ :

$$
\begin{gathered}
\operatorname{Per}\left(E_{t}, \Omega\right)+\int_{E_{t}}(t-f(x)) d x \leq \operatorname{Per}\left(E_{t} \backslash\left(W_{\varepsilon}-(\delta+\varepsilon) e\right), \Omega\right) \\
+\int_{E_{t} \backslash\left(W_{\varepsilon}-(\delta+\varepsilon) e\right)}(t-f(x)) d x \\
\operatorname{Per}\left(E_{s}, \Omega\right)+\int_{E_{s}}(s-f(x)) d x \leq \operatorname{Per}\left(E_{s} \cup W_{\varepsilon}, \Omega\right) \\
+\int_{E_{s} \cup W_{\varepsilon}}(s-f(x)) d x .
\end{gathered}
$$

Now, if we let

$$
L_{t}=\mathcal{H}^{N-1}\left(\partial W_{\varepsilon} \backslash \tilde{\partial} E_{s}\right) \quad \text { and } \quad L_{s}=\mathcal{H}^{N-1}\left(\partial W_{\varepsilon} \cap \tilde{\partial} E_{s}\right),
$$

we have that

$$
\operatorname{Per}\left(E_{t} \backslash\left(W_{\varepsilon}-(\delta+\varepsilon) e\right), \Omega\right)=\operatorname{Per}\left(E_{t}, \Omega\right)-L_{t}+L_{s}
$$

and

$$
\operatorname{Per}\left(E_{s} \cup W_{\varepsilon}, \Omega\right)=\operatorname{Per}\left(E_{s}, \Omega\right)+L_{t}-L_{s},
$$

so that, summing both equations in (5.3), we deduce

$$
\int_{W_{\varepsilon}-(\delta+\varepsilon) e}(t-f(x)) d x \leq \int_{W_{\varepsilon}}(s-f(x)) d x .
$$

Hence,

$$
(t-s)\left|W_{\varepsilon}\right| \leq \int_{W_{\varepsilon}}(f(x+(\delta+\varepsilon) e)-f(x)) d x \leq\left|W_{\varepsilon}\right| \omega_{f}(\delta+\varepsilon) .
$$


Dividing both sides by $\left|W_{\varepsilon}\right|>0$ and sending then $\varepsilon$ to zero, we deduce

$$
t-s \leq \omega_{f}(\delta) \text {. }
$$

The regularity of $u$ follows, that is, $\omega_{u} \leq \omega_{f}$. Now, if $f$ is continuous in $\bar{\Omega}$ we may approximate it uniformly by smooth functions $f_{\epsilon} \in C^{\infty}(\bar{\Omega})$. If $u_{\epsilon}$ is the corresponding solution of (1.4) with Neumann boundary condition, then we already proved that $\omega_{u_{\epsilon}} \leq \omega_{f_{\epsilon}}$. In particular, this gives us the equicontinuity of $u_{\epsilon}$. By uniqueness of solutions of the problem (1.4) with Neumann boundary condition, we have that $u_{\epsilon} \rightarrow u$ in $C(\bar{\Omega})$ where $u$ is the solution corresponding to $f$ and we get that $\omega_{u} \leq \omega_{f}$.

Finally, if $\Omega$ is an arbitrary convex subset of $\mathbb{R}^{N}$, we can approximate $\Omega$ by a sequence of smooth, strictly bounded uniformly convex sets $\Omega_{n}$ and consider $u_{n}$ the solution of (1.4) with homogeneous Neumann boundary condition in $\Omega_{n}$. Then, each $u_{n}$ is uniformly continuous with $\omega_{u_{n}} \leq \omega_{f}$. Passing to the limit and recalling the uniqueness of the Neumann solution $u$ of (1.4), we get the thesis.

Let us recall some basic definitions in order to state the analogous of Theorem 5.1 for the total variation flow. For brevity, we shall only sketch the results. As above, we assume that $\Omega$ is an open convex set in $\mathbb{R}^{N}$. Let us consider the minimizing total variation flow

$$
\begin{array}{ll}
\frac{\partial u}{\partial t}=\operatorname{div}\left(\frac{D u}{|D u|}\right) & \text { in } \left.Q_{T}=\right] 0, T[\times \Omega, \\
\frac{D u}{|D u|} \cdot \nu_{\Omega}=0 & \text { in } \left.Q_{T}=\right] 0, T[\times \partial \Omega
\end{array}
$$

with the initial condition

$$
u(0, x)=f(x), \quad x \in \Omega .
$$

Here equation (5.4) is formal and should not, for instance, be interpreted in the sense of viscosity solutions, but rather as the semigroup of an appropriately defined subgradient of the total variation. In particular, in the Hilbertian framework (in $L^{2}$ ), it is simply the gradient flow of the total variation, as defined in [10]. In the general case we shall follow $[5,8,11]$ (where the case of the total variation flow in unbounded domains is considered).

We define the operator $\mathcal{A}_{p}$ in $L^{p}(\Omega), \frac{N}{N-1} \leq p \leq \infty$, by

$$
v \in \mathcal{A}_{p} u \text { if and only if } u, v \in L^{p}(\Omega), u \in B V(\Omega) \text {, and }
$$

there exists $z \in X(\Omega)_{p}$ with $\|z\|_{\infty} \leq 1$ such that

$$
(z \cdot D u)=|D u|, \quad\left[z \cdot \nu_{\Omega}\right]=0, \quad \text { and } v=-\operatorname{div}(z) \quad \text { in } \mathcal{D}^{\prime}(\Omega) .
$$


Proposition 5.2. $[5,8,11]$ The operator $\mathcal{A}_{p}$ is m-accretive in $L^{p}(\Omega)$; that is, for any $f \in L^{p}(\Omega)$ and any $\delta>0$ there is a unique solution $u \in L^{p}(\Omega)$ of the problem

$$
u+\delta \mathcal{A}_{p} u \ni f
$$

Moreover, if $u_{1}, u_{2} \in L^{p}(\Omega)$ are the solutions of (5.6) corresponding to the right-hand sides $f_{1}, f_{2} \in L^{p}(\Omega)$, then

$$
\left\|u_{1}-u_{2}\right\|_{p} \leq\left\|f_{1}-f_{2}\right\|_{p}
$$

Moreover, the domain of $\mathcal{A}_{p}$ is dense in $L^{p}(\Omega)$ when $p<\infty$ and its closure contains $B U C(\bar{\Omega})$ (the space of bounded uniformly continuous functions) when $p=\infty$.

We denote by $\mathcal{R}_{\delta} f$ the solution of (5.6), and by $\mathcal{R}_{\delta}^{k} f$ its $k$-iterate, for any $k \geq 1$. By Proposition 5.2 and Crandall-Ligget's semigroup generation theorem [15], the following limit exists

$$
S(t) f:=\lim _{\delta \downarrow 0, k \delta \rightarrow t} \mathcal{R}_{\delta}^{k} f \in C\left([0, T], L^{p}(\Omega)\right)
$$

and is the semigroup solution of (5.4). Let us recall is characterization it in more classical terms.

By $L_{w}^{1}(] 0, T[; B V(\Omega))$ we denote the space of weakly* measurable functions $w:[0, T] \rightarrow B V(\Omega)$ (i.e., $t \in[0, T] \rightarrow\langle w(t), \phi\rangle$ is measurable for any $\phi$ in the predual of $B V(\Omega))$ such that $\int_{0}^{T}\|w(t)\| d t<\infty$.

Definition 5.3. A function $u \in C\left([0, T] ; L^{p}(\Omega)\right)$ is a strong solution of (5.4) if

$$
u \in W_{l o c}^{1,1}\left(0, T ; L^{p}(\Omega)\right) \cap L_{w}^{1}(] 0, T[; B V(\Omega))
$$

and there exists $z \in L^{\infty}(] 0, T\left[\times \Omega ; \mathbb{R}^{N}\right)$ with $\|z\|_{\infty} \leq 1$ such that

$$
\begin{gathered}
\int_{\Omega}(z(t) \cdot D u(t))=\int_{\Omega}|D u(t)| \quad \text { for a.e. } t>0, \\
{\left[z(t) \cdot \nu_{\Omega}\right]=0 \quad \text { in } \partial \Omega \text { for a.e. } t>0,}
\end{gathered}
$$

and

$$
u_{t}=\operatorname{div} z \quad \text { in } \mathcal{D}^{\prime}(] 0, T[\times \Omega) .
$$

We use the same definition if we replace $L^{p}(\Omega)$ by $B U C(\bar{\Omega})$. 
Theorem 5.4. $[5,8]$ Let $f \in L^{p}(\Omega)$ if $\frac{N}{N-1} \leq p<\infty$, or let $f \in B U C(\bar{\Omega})$ if $p=\infty$. Then

$$
u(t)=S(t) f:=\lim _{\delta \downarrow 0, k \delta \rightarrow t} \mathcal{R}_{\delta}^{k} f \in C\left([0, T], L^{p}(\Omega)\right)
$$

is a strong solution of (5.4). Moreover, strong solutions are unique.

In particular, strong and semigroup solutions of (5.4) coincide.

Remark 5.5. Notice that given $p \in\left[\frac{N}{N-1}, \infty\right]$ the $\operatorname{limit}_{\lim _{\delta \downarrow 0, k \delta \rightarrow t}} \mathcal{R}_{\delta}^{k} f$ is taken in $L^{p}(\Omega)$.

Let us point out that a more general existence and uniqueness result of solutions of $(5.4)$ for initial data in $L_{l o c}^{p}(\bar{\Omega})$ for any $p \in[1, \infty]$ holds $[8,11]$. Indeed, it is proved in $\mathbb{R}^{N}$ in $[8,11]$ but it can be easily adapted to the case of Neumann boundary condition in $\bar{\Omega}$ using the techniques in [4].

As a consequence of Theorems 5.1 and 5.4, we deduce:

Corollary 5.6. Let $f$ be a uniformly continuous function in $\bar{\Omega}$ and $u(t, x)$ be the Total Variation flow starting from $f$, with Neumann boundary condition. Then, for any $t \geq 0, u(t, \cdot)$ is uniformly continuous with modulus $\omega_{u(t, \cdot)} \leq \omega_{f}$.

Proof. If $f \in B U C(\bar{\Omega})$, this is a consequence of Theorem 5.1 and Theorem 5.4. If we only assume that $f$ is uniformly continuous in $\bar{\Omega}$, we may approximate it by functions in $B U C(\bar{\Omega})$, apply the result in this case, and use the uniqueness result for data in $L_{l o c}^{\infty}(\bar{\Omega})$ mentioned before the statement of the corollary.

\section{References}

[1] Almgren, F., Taylor, J. And Wang, L.-H.: Curvature-driven flows: a variational approach. SIAM J. Control Optim. 31 (1993), no. 2, 387-438.

[2] Alter, F., Caselles, V. and Chambolle, A.: A characterization of convex calibrable sets in $\mathbb{R}^{N}$. Math. Ann. 332 (2005), no. 2, 329-366.

[3] Ambrosio, L., Fusco, N. And Pallara, D.: Functions of bounded variation and free discontinuity problems. Oxford Mathematical Monographs. The Clarendon Press Oxford University Press, New York, 2000.

[4] Andreu, F., Ballester, C., Caselles, V. and Mazón, J. M.: The Dirichlet problem for the total variation flow. J. Funct. Anal. 180 (2001), no. 2, 347-403.

[5] Andreu, F., Caselles, V. And Mazón, J. M.: Parabolic quasilinear equations minimizing linear growth functionals. Progress in Mathematics 223. Birkhäuser Verlag, Basel, 2004. 
[6] Anzellotti, G.: Pairings between measures and bounded functions and compensated compactness. Ann. Mat. Pura Appl. IV 135 (1983), 293-318.

[7] Barozzi, E., Gonzalez, E. and Tamanini, I.: The mean curvature of a set of finite perimeter. Proc. Amer. Math. Soc. 99 (1987), no. 2, 313-316.

[8] Bellettini, G., Caselles, V. and Novaga, M.: The total variation flow in $\mathbb{R}^{N}$. J. Differential Equations 184 (2002), no. 2, 475-525.

[9] Bellettini, G., Caselles, V. and Novaga, M.: Explicit solutions of the eigenvalue problem $-\operatorname{div}\left(\frac{D u}{|D u|}\right)=u$ in $\mathbb{R}^{2}$. SIAM J. Math. Anal. 36 (2005), no. 4, 1095-1129.

[10] Brézis, H.: Opérateurs maximaux monotones et semi-groupes de contractions dans les espaces de Hilbert. North-Holland Math. Stud. 5, Notas Mat. 50. North-Holland, Amsterdam-London; American Elsevier, New York, 1973.

[11] Caselles, V. and Chambolle, A.: Anisotropic curvature-driven flow of convex sets. Nonlinear Anal. 65 (2006), no. 8, 1547-1577.

[12] Caselles, V., Chambolle, A. and Novaga, M.: The discontinuity set of solutions of the TV denoising problem and some extensions. Multiscale Model. Simul. 6 (2007), no. 3, 879-894.

[13] Chambolle, A. And Lions, P.-L.: Image recovery via total variation minimization and related problems. Numer. Math. 76 (1997), no. 2, $167-188$.

[14] Chan, T., Golub, G.H. And Mulet, P.: A nonlinear primal-dual method for total variation-based image restoration. SIAM J. Sci. Comput. 20 (1999), no. 6, 1964-1977.

[15] Crandall, M. G. and Liggett, T. M.: Generation of semi-groups of nonlinear transformations on general Banach spaces. Amer. J. Math. 93 (1971), 265-298.

[16] Federer, H.: Geometric measure theory. Die Grundlehren der mathematischen Wissenschaften 153. Springer-Verlag, New York, 1969.

[17] Gilbarg, D. and Trudinger, N.S.: Elliptic partial differential equations of second order. Classics in Mathematics. Springer-Verlag, Berlin, 2001.

[18] Massari, U.: Esistenza e regolarità delle ipersuperfice di curvatura media assegnata in $\mathbb{R}^{n}$. Arch. Rational Mech. Anal. 55 (1974), 357-382.

[19] Massari, U.: Frontiere orientate di curvatura media assegnata in $L^{p}$. Rend. Sem. Mat. Univ. Padova 53 (1975), 37-52.

[20] Massari, U. and Pepe, L.: Successioni convergenti di ipersuperfici di curvatura media assegnata. Rend. Sem Mat. Univ. Padova 53 (1975), 53-68.

[21] PAolini, E.: Regularity for minimal boundaries in $\mathbb{R}^{n}$ with mean curvature in $L^{n}$. Manuscripta Math. 97 (1998), no. 1, 15-35. 
[22] Rudin, L., Osher S. J. And Fatemi, E.: Nonlinear total variation based noise removal algorithms. Phys. D. 60 (1992), 259-268.

[23] Tamanini, I.: Boundaries of Caccioppoli sets with Hölder-continuous normal vector. J. Reine Angew. Math. 334 (1982), 27-39.

Recibido: 8 de abril de 2009

Revisado: 26 de octubre de 2009

Vicent Caselles

Departament de Tecnologies de la Informació i les Comunicacions

Universitat Pompeu Fabra

C/Roc Boronat 138

08018 Barcelona, Spain

vicent.caselles@upf.es

Antonin Chambolle

CMAP, Ecole Polytechnique, CNRS

91128 Palaiseau, France

antonin.chambolle@polytechnique.fr

Matteo Novaga

Dipartimento di Matematica

Università di Padova

Via Trieste 63

35121 Padova, Italy

novaga@math. unipd.it

\footnotetext{
V. Caselles acknowledges partial support by GRC, reference 2009 SGR 773, and by "ICREA Acadèmia" prize for excellence in research, both funded by the Generalitat de Catalunya, and by PNPGC project, reference MTM2006-14836. A. Chambolle is partially supported by the CNRS, and by the Agence Nationale de la Recherche, grant ANR-08BLAN-0082.
} 